\title{
The Annual Trends between Neurointerventional and Neurosurgical Procedures in Korea: Analysis using HIRA Data from 2010 to 2016
}

\author{
Sang Hyun Suh, MD ${ }^{1,2}$
}

Purpose: The purpose of this study was to evaluate the annual trend for several neurosurgical and neurointerventional procedures (NIPs) in Korea between 2010 and 2016 by using medical claim data.

Materials and Methods: All data during the recent 7 years were collected from Bigdata Hub provided by the Health Insurance Review \& Assessment Service (HIRA). Using several codes for medical practice, we estimated the number of in-patients for each practice and calculated the annual variation of each procedure. These data were compared between each NIP versus the equivalent surgical procedure and analyzed according to the geographic distribution and the hospital grade.

Results: During the past 7 years, the average growth rate of all stroke in-patients is $2.05 \%$, and the number of in-patients with ischemic stroke is 5.2 times higher than those with hemorrhagic stroke. While clipping accounted for $53.7 \%(5,209 / 9,700)$ of total patients in $2010,61.9 \%$ of patients $(9,146 / 14,781)$ are currently undergoing coiling procedure in 2016 . MT for acute ischemic stroke has grown annually by an average of $35.9 \%$ during the past 3 years. Stenting and angioplasty for carotid artery and intracranial artery showed relatively stable growth during the past 7 years. Especially, carotid artery stenting is rapidly increasing in secondary general hospitals, where more than $50 \%$ of these procedures were performed after 2014 .

Conclusion: In this study, we found that most of NIPs has increased annually over the past 7 years and that NIPs are rapidly replacing conventional surgical maneuvers in Korea.

Key Words : Hospital; Korea; Health insurance; Stroke; Aneurysm; Carotid artery

'Department of Radiology, Gangnam Severance Hospital, Yonsei University College of Medicine, Seoul, Korea 2Severance Institute of Vascular and Metabolic Research, Yonsei University College of Medicine, Seoul, Korea Received July 9, 2017; Revised July 18, 2017; Accepted July 18, 2017.

Correspondence to: Sang Hyun Suh, MD, PhD, Department of Radiology, Gangnam Severance Hospital, Yonsei University College of Medicine, 211 Eonju-ro, Gangnam-gu, Seoul 06273, Korea.

Tel. 82.2.2019.3510 Fax. 82.2.3462.5472 E-mail: suhsh11@yuhs.ac

This is an Open Access article distributed under the terms of the Creative Commons Attribution Non-Commercial License (http://creativecommons.org/licenses/by-nc/3.0) which permits unrestricted non-commercial use, distribution, and reproduction in any medium, provided the original work is properly cited. 


\section{Sang Hyun Suh}

Since introduction of coil embolization for an intracranial aneurysm as a neurointerventional procedure (NIP) in Korea, NIPs have grown into a major role for the treatment of head and neck vascular disease during the past 23 years. The field of NIP includes not only cerebral aneurysm, but also intra- and extracranial arterial stenosis, acute ischemic stroke, vascular diseases in the head and neck, and spinal cord vascular diseases. Along with expansion of the NIP field, the number of specialized institutions and specialists has increased greatly, and related academic societies have continued to grow qualitatively and quantitatively.

In Korea, the Health Insurance Review \& Assessment Service (HIRA), being established in July 2000, is responsible for reviewing medical fees as well as assessing quality of health care services provided to health insurance beneficiaries. They analye and monitor 1.4 billion medical service cases provided by 85,000 providers annually. Like other medical practices, most of the NIPs are covered by medical insurance benefits, which makes it possible to obtain insurance information data for each NIP practice.

Recently HIRA has developed a medical big data system and opened public data for healthcare service as "Big data Hub (http://opendata.hira.or.kr/ )" on 2015 $[1,2]$. Using these online data, we tried to evaluate the nationwide trend for each NIP according to the geographic distribution and the grading of the hospital. In addition, we also attempted to compare data for several NIPs, including aneurysm coiling, carotid artery stenting (CAS), mechanical thrombectomy (MT) for acute ischemic stroke, stenting and angioplasty for intracranial artery (ICA) stenosis, with those for equivalent surgical procedures, such as aneurysm clipping or carotid endarterectomy (CEA).

\section{MATERIALS AND METHODS}

\section{Study data acquisition}

This study was approved by the Institutional Review Board at our hospital. The nationwide claims data of HIRA is collected when healthcare service providers submit a claim to HIRA to be reimbursed for a healthcare service that they provided to patients. All data in this study were obtained from Bigdata $\mathrm{Hub}$ (http://opendata.hira.or.kr/), which HIRA has recently developed as opened public data for healthcare service. We reviewed these data from Bigdata Hub for the number of the patients who had the specific codes between January 2010 and December 2016.

\section{Data collection and analysis}

The data of the HIRA was able to be classified by coding system and we collected data using several codes about "diagnosis" defined by the Korean Standard Classification of Diseases and "healthcare service" for each procedure or operation. Table 1 summarized several codes used for identification of samples in this study. For each year, we estimated the number of in-patients with any stroke, such as subarachnoid hemorrhage, hemorrhagic or ischemic stroke and calculated the annual frequency of each procedure (Table 1). In addition, we analyzed data according to the regional distribution and the hospital grade.

We compared the annual data between various practices, such as coiling versus clipping, simple versus assisted coiling or CAS versus CEA, and also estimated the annual variation of each procedure or operation.

Table 1. The Codes Used to Identify Samples from the Bigdata Hub of the HIRA

\begin{tabular}{lll}
\hline Files & Variables & Codes \\
\hline Diagnosis & Cerebrovascular disease & I60/161/l62/l63/l64 \\
\hline Healthcare services (in-patient procedures or operation) & Thrombolysis, cerebral vessels & M6631 \\
\cline { 2 - 3 } & Mechanical thrombolysis & S6633 \\
\cline { 2 - 3 } & Clipping, cerebral aneurysm & M1662/M1661 \\
\cline { 2 - 3 } & Coil embolization, cerebral aneurysm & M6593/M6594 \\
\cline { 2 - 3 } & Angioplasty, cerebral/carotid, vertebral arteries & O0226, O0227, O2066 \\
\cline { 2 - 3 } & Carotid endarterectomy & M6601/M6602 \\
\cline { 2 - 3 } & Stenting, cerebral/carotid, vertebral arteries & \\
\hline
\end{tabular}




\section{Nationwide Trends of Korean Neurointervention}

\section{RESULTS}

\section{Nationwide distribution of in-patients diagnosed} with Stroke over the past 7 years

From 2010 to 2016, the average annual number of inpatients diagnosed with stroke was 558,051 in total, which consisted of 469,423 (84.1\%) in ischemic stroke and 88,627 (15.9\%) in hemorrhagic stroke (Fig. 1). The average number of in-patients with subarachnoid hemorrhage was 27,909 per year, which was one third of in-patients with hemorrhagic stroke. During the past 7 years, the annual growth rate of stroke in-patients is $2.05 \%$, and the number of in-patients with ischemic stroke was 5.2 times higher than those with hemorrhagic stroke.

\section{Aneurysm treatment; Clipping versus Coiling}

For cerebral aneurysm treatment, 9,700 patients were treated in 2010 and it increased to 14,781 in 2016.
While clipping accounted for $53.7 \%(5,209 / 9,700)$ of total patients in $2010,61.9 \%$ of patients $(9,146 / 14,781)$ has undergone coiling procedure in 2016. In particular, the number of all treated patients was increased markedly by $20.4 \%$ in 2016 , which is proportional to the increase in coiling patients. Figure 2 showed that coil embolization had played the major role in current management of intracranial aneurysms since 2012.

In coiling procedures, simple coiling accounted for more than $50 \%$ of all treated patients by 2015 , but assisted coiling procedure by stent or balloon has increased rapidly and has emerged as the main method to treat $51 \%$ of patients $(4,660 / 9,146)$ in 2016 . In simple coiling procedure, the average increase rate was $10.1 \%$ over the last 7 years, while the assisted coiling procedure increased by $16.2 \%$ on average, which showed treatment patterns of coil embolization have recently changed (Fig. 3).

In the geographical distribution, coil embolization
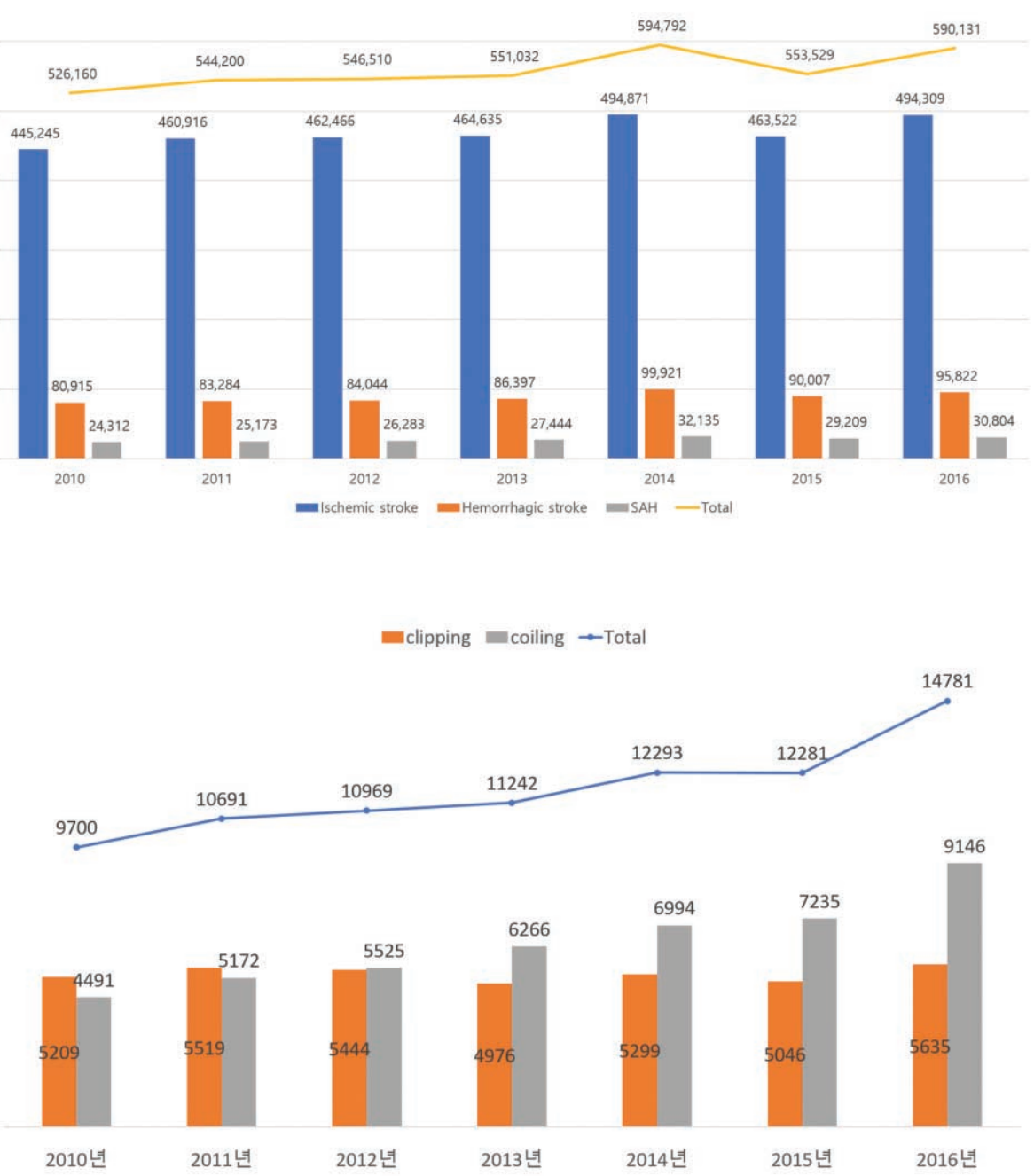

Fig. 1. The annual trends of in-patients with ischemic and hemorrhagic stroke, including subarachnoid hemorrhage (2010-2016).

Fig. 2. Comparison of annual trends between coiling and clipping (20102016). 


\section{Sang Hyun Suh}

accounted for $31.6 \%(1,985 / 6,410)$ in Seoul, $18.6 \%$ $(1,201 / 6,410)$ in Gyeonggi, $10.6 \%(688 / 6,410)$ in Busan, 6.6\% (424/6,410) in Daegu and 5.3\% $(342 / 6,410)$ in Daejeon. Although Seoul accounted for $1 / 3$ of the total coiling treatments, which decreased from $37.6 \%(1,688 / 4,494)$ in 2010 to $28.4 \%$ $(2,605 / 9,157)$ in 2016 , the Gyeonggi, Busan, Daegu and Daejeon have been still growing.

Coiling procedures in tertiary hospital were decreased from $67.6 \%(3,035 / 4492)$ in 2010 to $59.6 \%$ (5451/9152) in 2016, but opposite in secondary general hospital, ranging from $30.9 \%(1389 / 4492)$ in 2010 to $40.2 \%$ (3676/9152) in 2016.

\section{Acute ischemic stroke treatment; Mechanical thrombectomy}

After solitaire FR was registered as a healthcare insurance benefit for acute ischemic stroke treatment in August 2014, it has grown annually by an average of $35.9 \%$ during the recent 3 years and is expected to increase to more than 2,700 by 2016 (Table 2).

\section{Carotid artery stenosis: Carotid artery stenting versus endarterectomy}

During the past 7 years, the number of patients who have undergone CEA is an average of 512.7 per yea, which changed from $19.7 \%(430 / 2183)$ in 2010 to $19 \%$

Table 2. Annual Trend of Mechanical Thrombectomy for AIS Treatment (2013-2016)

\begin{tabular}{lllll}
\hline Year & 2013 & 2014 & 2015 & 2016 (expected) \\
\hline MT & 1103 & 1633 & 2328 & 2723 \\
\hline
\end{tabular}

MT= mechanical thrombectomy
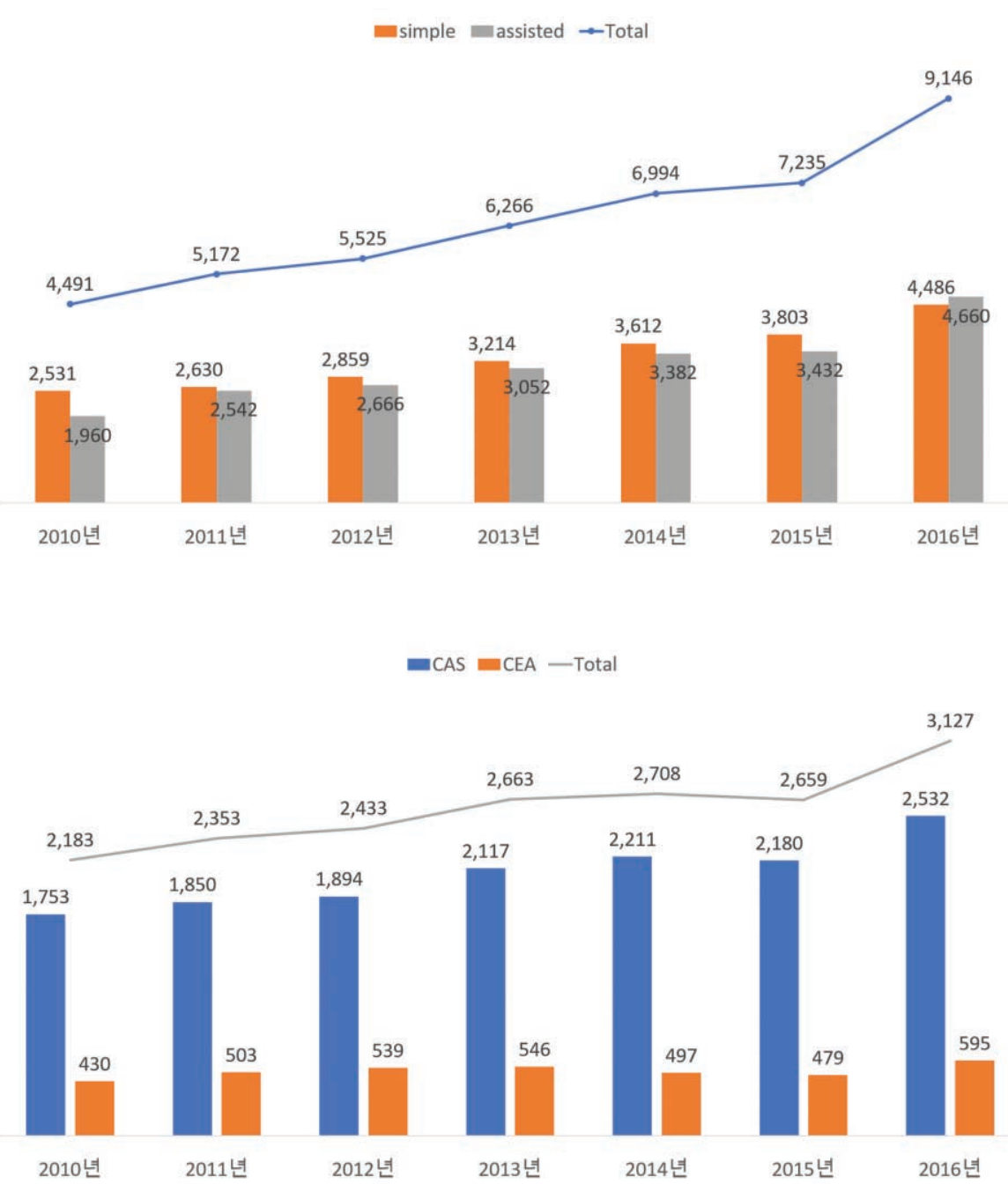

Fig. 3. The annual trend for the coiling procedure (2010-2016).

Fig. 4. The annual trends between CAS and CEA (2010-2016). 


\section{Nationwide Trends of Korean Neurointervention}

$(595 / 3127)$ in 2016 . CAS was performed in an average of 2,076 per year, ranging from $80.3 \%(1753 / 2183)$ in 2010 to $81 \%(2532 / 3127)$ in 2016 (Fig. 4).

CAS was performed in an average of $47.5 \%$ in Seoul and Gyeonggi, and $13.4 \%$ in Busan and Gyeongnam, 9.9\% in Daejeon, Chungnam, and 9.1\% in Daegu and Gyeongbuk. In particular, the frequency of CAS has been recently increasing in hospitals of Gyeonggi and Chungnam. Although there was no significant change in the number of procedures in tertiary hospitals since 2010, CAS was rapidly increasing in secondary general hospitals, where more than $50 \%$ of all CAS procedure was performed after 2014.

CEA was treated in Seoul by an average of $49.7 \%$, $11.6 \%$ in Daegu, $10.4 \%$ in Gwangju, $6.6 \%$ in Gyeonggi and $5.9 \%$ in Busan. Most CEA procedures were performed in tertiary hospitals.

\section{Intracranial artery stenosis: stenting versus angioplasty}

Stenting and angioplasty for intracranial artery stenosis was performed in an average of 756 patients per year. Intracranial stenting has markedly decreased in 2013 , but it has been gradually increasing since 2016. Intracranial angioplasty had an average of 200 procedures annually for the past 7 years (Fig. 5).

\section{DISCUSSION}

This study demonstrates most NIPs in Korea have increased in tendency over the past 7 years. Especially, coiling procedure for aneurysm treatment shows a steep increase in the number of NIPs in 2016, being close to twice the number of clipping. The reasons for the increase in coiling procedures are (1) the widespread application of the coiling procedure due to advancement in treatment materials and techniques and (2) promotion of coil surgery in secondary general hospitals. Recent data indicate that assisted coil embolization using stent or balloon is more common than a simple coiling procedure, and the assisted coiling technique accounted for more than $50 \%$ of all coiling treatments in 2016. During the past 7 years, the average growth rate of aneurysm coiling in the tertiary hospital is $10.6 \%$; however, the average rate at secondary general hospitals is $18 \%$, which means the increase in coil procedures at secondary general hospitals is likely to play a major role in the increase of all coil procedures (Table 3 ).

The AIS treatment strategy had a major turning point in 2015 with several randomized clinical trials (RCT) results [3-6], and MT procedures in Korea has been expected to increase annually by about $30 \%$. The

Table 3. Annual Trend of In-Patients with Aneurysm Coiling According to the Hospital Classification (2010-2016)

\begin{tabular}{lrrrrrrrr}
\hline & 2010 & 2011 & 2012 & 2013 & 2014 & 2015 & 2016 \\
\hline Tertiary hospital & 3,035 & 3,225 & 3,270 & 3,585 & 4,206 & 4,309 & 5,451 \\
Secondary hospital & 1,389 & 1,849 & 2,145 & 2,610 & 2,791 & 2,926 & 3,676 \\
Primary hospital & 68 & 100 & 114 & 73 & 0 & 5 & 25 \\
Total & 4,492 & 5,174 & 5,529 & 6,268 & 6,997 & 7,240 & 9,152 \\
\hline
\end{tabular}

$$
\text { aICA stent }=\text { ICA PTA -Total }
$$

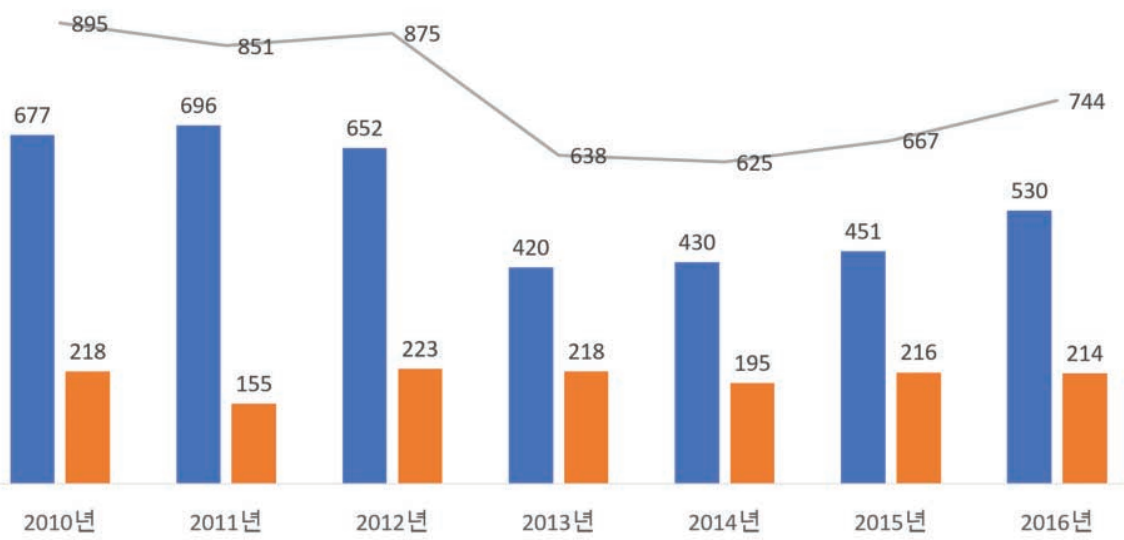

Fig. 5. The annual trends for intracranial stents and angioplasty (2010-2016). 


\section{Sang Hyun Suh}

reason for the increase in MT procedures is thought to be application of the healthcare reimbursement for MT since August 2014 and possibility of expanding treatment indications for MT from the next RCT.

CAS showed a below-average growth rate except in 2013 and 2016, with an average growth rates of more than $10 \%$. The reason for low growth of the CAS procedure may be that the indication for CAS procedures has been more strictly applied with the announcement of two RCT results [7, 8] since 2006. Interestingly, the number of CEA procedures performed in Korea has not changed much over the past 7 years, and the number of CAS procedures performed by tertiary hospitals is almost constant. An increasing trend of CAS in secondary hospitals means that CAS is becoming the common procedure among clinicians.

After SAMMPRIS trial [9], stenting and angioplasty of ICA in Korea has decreased since 2012. In particular, ICA stenting procedures has decreased markedly by $36 \%$ in 2013. However, in the past 3 years, the number of these procedures have gradually increased, which may be related to the high prevalence of intracranial artery stenosis in Korea.

The limitations of this study are as follows: (1) The data used in this study were collected only from the online contents, which were provided only from 2010 and 2016; (2) There is a possibility that some contents may be duplicated or missed due to the insufficient segmentation of "medical practice" codes, which were used as keywords; (3) subgroup analysis was not available because the cross-searching between codes of "diagnosis" and "medical practice" was impossible.

\section{CONCLUSION}

This study demonstrated that most NIPs have increased annually between 2010 and 2016 and that these procedures are rapidly replacing existing surgical maneuvers. Although advances in technology and materials have made NIPs more common throughout the country, there is a need for aggressive measures by our academic society to solve some problems such as geographic imbalance of the special institutions and a lack of quality management for specialists.

\section{Acknowledgments}

This study was supported by the Korean Society of Interventional Neuroradiology (KSIN) research grant 2016. The author appreciates Cho, Young Taek (Codman Neurovascular, Korea) and An, Bosun (Medtronic, Korea) for their help.

\section{References}

1. Kim L, Kim JA, Kim S. A guide for the utilization of Health Insurance Review and Assessment Service National Patient Samples. Epidemiol Health 2014;36:e2014008

2. Kim JA, Yoon S, Kim LY, Kim DS. Towards actualizing the value potential of korea health insurance review and assessment (hira) data as a resource for health research: Strengths, limitations, applications, and strategies for optimal use of hira data. $J$ Korean Med Sci 2017;32:718-728

3. Fransen PS, Beumer D, Berkhemer OA, van den Berg LA, Lingsma $\mathrm{H}$, van der Lugt A, et al. MR CLEAN, a multicenter randomized clinical trial of endovascular treatment for acute ischemic stroke in the netherlands: study protocol for a randomized controlled trial. Trials 2014;15:343

4. Goyal M, Demchuk AM, Menon BK, Eesa M, Rempel JL, Thornton J, et al. Randomized assessment of rapid endovascular treatment of ischemic stroke. N Engl J Med 2015;372:1019-1030

5. Saver JL, Goyal M, Bonafe A, Diener HC, Levy EI, Pereira VM, et al. Stent-retriever thrombectomy after intravenous t-pa vs. T-pa alone in stroke. N Engl J Med 2015;372:2285-2295

6. Campbell BC, Mitchell PJ, Kleinig TJ, Dewey HM, Churilov L, Yassi N, et al. Endovascular therapy for ischemic stroke with perfusion-imaging selection. N Engl J Med 2015;372:1009-1018

7. Mas JL, Chatellier G, Beyssen B, Branchereau A, Moulin T, Becquemin JP, et al. Endarterectomy versus stenting in patients with symptomatic severe carotid stenosis. N Engl J Med 2006; 355:1660-1671

8. Group SC, Ringleb PA, Allenberg J, Bruckmann H, Eckstein HH, Fraedrich G, et al. 30 day results from the SPACE trial of stentprotected angioplasty versus carotid endarterectomy in symptomatic patients: a randomised non-inferiority trial. Lancet 2006; 368:1239-1247

9. Chimowitz MI, Lynn MJ, Derdeyn CP, Turan TN, Fiorella D, Lane BF, et al. Stenting versus aggressive medical therapy for intracranial arterial stenosis. $N$ Engl J Med 2011;365:993-1003 